\title{
PESCARIAS INDUSTRIAIS DO CAMARÃO-ROSA E DA FAUNA ACOMPANHANTE NO ESTADO DO RIO DE JANEIRO, BRASIL (1993 - 1997)
}

\author{
Pink-shrimp industrial fishery and its by-catch off \\ Rio de Janeiro State, Brazil (1993 - 1997)
}

\author{
Melquíades Pinto Paiva ${ }^{1,3}$, Antônio Alberto da Silveira Menezes², \\ Magda Fernandes de Andrade-Tubino ${ }^{1,4}$,
}

\begin{abstract}
RESUMO
As pescarias do camarão-rosa no Sudeste/Sul do Brasil ocorrem desde a foz do rio Doce (Estado do Espírito Santo) até o estuário da lagoa dos Patos (Estado do Rio Grande do Sul), alcançando as espécies Farfantepenaeus brasiliensis (Latreille, 1817) e Farfantepenaeus paulensis (Pérez Farfante, 1967), que são pescadas em conjunto e convivem nos mesmos pesqueiros. Neste trabalho tratamos das pescarias industriais do camarão-rosa e da sua fauna acompanhante, no Estado do Rio de Janeiro (1993 - 1997), com base nos registros dos mapas-de-bordo da frota camaroneira. As capturas do camarão-rosa decresceram a partir de 1994, o mesmo não acontecendo com a fauna acompanhante; as médias anuais foram 162,7 t para o camarão-rosa e 2.444,2 t para a fauna acompanhante. Para cada tonelada capturada do camarãorosa foram desembarcadas 15,0 t de fauna acompanhante; o aproveitamento desta cresce com a queda de produção do camarão-rosa. As capturas do camarão-rosa se concentram em junho - outubro, perfazendo o total de $74,6 \%$ da produção anual. Na composição em peso dos desembarques da fauna acompanhante, 0,8\% corresponderam aos crustáceos, 8,3\% aos moluscos e 90,9\% aos peixes. As médias da produtividade das pescarias do camarão-rosa foram $25 \mathrm{~kg} / \mathrm{dia}=7 \mathrm{~kg} /$ lance $=2 \mathrm{~kg} /$ hora; para a fauna acompanhante, $396 \mathrm{~kg} /$ dia $=108 \mathrm{~kg} / \mathrm{lance}=26 \mathrm{~kg} / \mathrm{hora}$. A queda da abundância relativa do camarão-rosa foi bem evidente a partir de 1994.
\end{abstract}

Palavras-chaves: camarão-rosa, pesca industrial, fáuna acompanhante, Rio de Janeiro.

\begin{abstract}
The pink-shrimp fisheries in Southeast/South Brazil occur from the mouth of Doce River (Espirito Santo State) to the estuary of Patos Lagoon (Rio Grande do Sul State), reaching the species Farfantepenaeus brasiliensis (Latreille, 1817) and Farfantepenaeus paulensis (Pérez Farfante, 1967), caught together and living on same fishing grounds. In this paper we deal with the pink-shrimp industrial fisheries and its by-catch at Rio de Janeiro State (1993 - 1997), based on fishing log-books of shrimp-trawlers. The pink-shrimp catches decreased from 1994, the same not occurring with the by-catch landings; the annual mean catches were $162.7 t$ for pink-shrimp and 2,444.2 $t$ for by-catch. For each pinkshrimp ton caught were landed $15.0 t$ of by-catch; its landings increases when the pink-shrimp production decreases. Pink-shrimp catches are concentrated in June - October, reaching $74.6 \%$ of the annual production. In the weight composition of by-catch landings, $0.8 \%$ corresponded to crustaceans, $8.3 \%$ to molluscs, and $90.9 \%$ to fishes. The yearly mean productivity of pink-shrimp fisheries were $25 \mathrm{~kg} /$ day $=7 \mathrm{~kg} / \mathrm{haul}=2 \mathrm{~kg} / \mathrm{hour} ;$ for by-catch, $396 \mathrm{~kg} / \mathrm{day}=108$ $\mathrm{kg} / \mathrm{haul}=26 \mathrm{~kg} / \mathrm{hour}$. The downward trend of pink-shrimp relative abundance is quite evident since 1994 .
\end{abstract}

Key words: pink shrimp, industrial fisheries, by-catch, Rio de Janeiro State (Brazil).

\footnotetext{
${ }_{1}^{1}$ Departamento de Biologia Marinha, Instituto de Biologia, Universidade Federal do Rio de Janeiro, Cidade Universitária, Rio de Janeiro-RJ, 21944-970. E-mail:mappaiva@uol.com.br

${ }^{2}$ Instituto Brasileiro do Meio Ambiente e dos Recursos Naturais Renováveis/Superintendência Estadual do Rio de Janeiro, Praça XV de Novembro, 42/3․ andar, Rio de Janeiro-RJ, 20010-010.

${ }^{3}$ Bolsista do Conselho Nacional de Desenvolvimento Científico e Tecnológico (CNPq).

${ }^{4}$ Bolsista da Fundação Carlos Chagas Filho de Amparo à Pesquisa do Estado do Rio de Janeiro (FAPERJ).
} 


\section{INTRODUÇÃO}

É bem antiga a explotação camaroneira ao longo da costa das regiões sudeste e sul do Brasil, onde são capturadas pelo menos sete espécies (Paiva, 1997). As mais importantes destas espécies, do ponto de vista econômico, são as vulgarmente conhecidas por camarão-rosa, pescadas em conjunto (Pérez Farfante \& Kensley, 1997; Zenger Jr. \& Agnes, 1977): Farfantepenaeus brasiliensis (Latreille, 1817), que ocorre desde a Carolina do Norte (Estados Unidos da América), Bermudas e Índias Ocidentais até o Rio Grande do Sul (Brasil); Farfantepenaeus paulensis (Pérez Farfante, 1967), encontrada do cabo de São Tomé - norte do estado do Rio de Janeiro (Brasil) até às proximidades de Mar del Plata (Argentina).

As pescarias do camarão-rosa no Sudeste/Sul do Brasil se realizam desde o estado do Espírito Santo, em frente à foz do rio Doce, até o estuário da lagoa dos Patos (estado do Rio Grande do Sul).

Nos anos de 1964 - 1994, a produção do camarão-rosa alcançou a média anual de 7.564 t (39,5\%), no conjunto dos desembarques controlados da explotação camaroneira, nas regiões sudeste e sul do Brasil; as capturas do camarão-rosa foram feitas pelos sistemas de produção artesanal $(59,0 \%)$ e industrial $(41,0 \%)$; os desembarques anuais controlados do camarão-rosa, no estado do Rio de Janeiro, atingiram as médias de $698 \mathrm{t}$ (78,6\%) pelo sistema industrial, e apenas 190 t (21,4\%) pelo sistema artesanal (Paiva, 1997).

Os estoques das espécies do camarão-rosa estão em processo de gradativo declínio, por causa da sobrepesca dos adultos e do aumento das capturas nos criadouros de ambas as espécies (D'Incao et al., 1995; Paiva, 1997).

Em todo o mundo, o principal problema ambiental da pesca industrial dos camarões reside na captura de uma fauna acompanhante constituída, em grande parte, de formas jovens da maioria das espécies que constituem sua biocenose, no desperdício econômico dessa biomassa e na agressão ao substrato ocasionada pela utilização de redes-de-arrasto, comprometendo a sustentabilidade das pescarias.

A participação da fauna acompanhante, nos desembarques do camarão-rosa no Estado de Santa Catarina (1989 - 1992), mostra uma grande predominância dos peixes (ósseos + cartilaginosos + mistura), perfazendo o total de $74,8 \%$, sendo os moluscos responsáveis por $22,1 \%$ e os crustáceos por $5,5 \%$ do peso registrado; a relação encontrada foi de $1 \mathrm{~kg}$ de camarão-rosa : $13 \mathrm{~kg}$ de fauna acompanhante (Kotas, 1995).

No litoral norte do Estado de São Paulo, cinco espécies de peixes se destacam nas capturas da fauna acompanhante do camarão-rosa (Vianna, 1998): mangangá-liso, Porichthys porosissimus (Valenciennes, 1837); coió, Dactylopterus volitans (Linnaeus, 1758); cabrinha, Prionotus punctatus (Bloch, 1797); cangauá, Ctenosciaena gracilicirrus (Metzelaar, 1919); e corcoroca, Orthopristis ruber (Cuvier, 1830). Os dados disponíveis indicam a depleção dos estoques destas espécies, com sobrepesca das suas populações, apesar das pescarias serem dirigidas para o camarão-rosa.

As causas determinantes da rejeição de pescado a bordo de camaroneiros engajados na explotação do camarão-rosa são as seguintes (Kotas, op. cit.): não aceitação de espécies no mercado consumidor, pequeno porte dos indivíduos capturados e problemas de conservação a bordo, por carência de gelo e/ou espaço em câmaras frigoríficas.

O presente trabalho trata das pescarias industriais do camarão-rosa e da fauna acompanhante, no Estado do Rio de Janeiro, Sudeste do Brasil (figura 1), nos anos de 1993 - 1997.

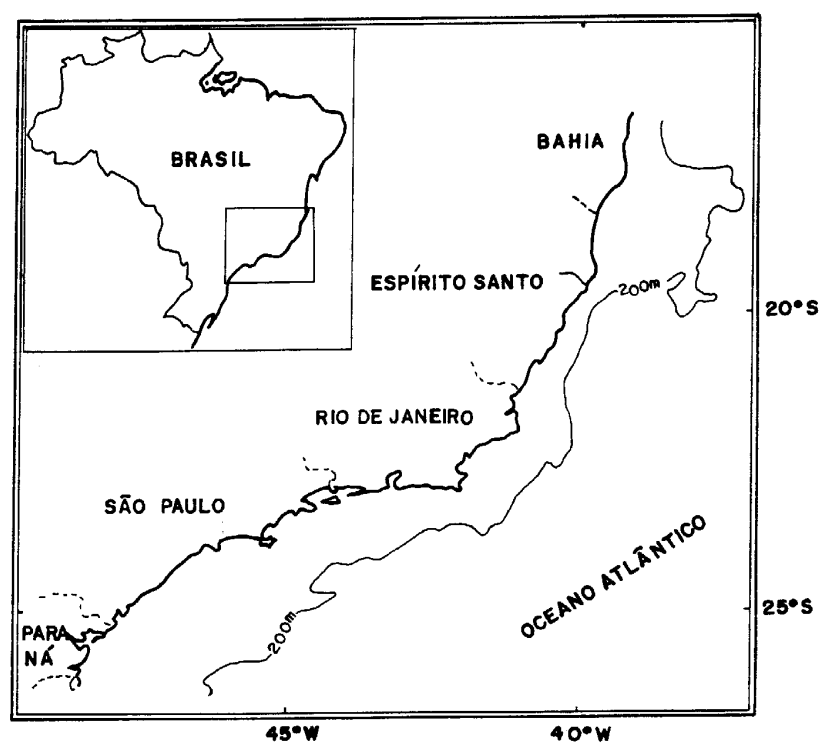

Figura 1 - Costa sudeste do Brasil, onde se encontra o Estado do Rio de Janeiro.

\section{MATERIAL E MÉTODOS}

Este trabalho se fundamenta em dados contidos nos mapas-de-bordo da frota industrial baseada em portos do Estado do Rio de Janeiro, engajada na explotação do camarão-rosa (1993 - 1997). Os dados anotados foram os seguintes: (a) desembarques mensais/anuais do camarão-rosa e das diferentes espécies que constituem a sua fauna acompanhante; (b)esforço de pesca anual aplicado pela frota, nas unidades "dia de pesca", "lance" e "hora de arrasto".

Os desembarques de espécies da fauna acompanhante formaram os agrupamentos de crustáceos, 
moluscos e peixes, possibilitando os cálculos de relações anuais das partes e do total com as capturas do camarão-rosa (Tabelas I e II). Também, procuramos conhecer os ciclos anuais dos desembarques do camarão-rosa e da fauna acompanhante, considerando os diferentes meses, com ou sem inclusão dos períodos de defeso (Tabelas III e IV). Especial atenção foi dada à composição da fauna acompanhante, para destacar a participação dos grupos e das suas espécies de maior interesse econômico (Tabela V).

Tabela I-Capturas desembarcadas do camarão-rosa e da fauna acompanhante resultantes de pescarias ao longo da costa do Estado do Rio de Janeiro (Brasil), nos anos de 1993 - 1997.

\begin{tabular}{l|c|c|c|c|c}
\hline \multirow{2}{*}{ Anos } & \multicolumn{5}{|c}{ Capturas desembarcadas (t) } \\
\cline { 2 - 6 } & camarão & \multicolumn{4}{|c}{ fauna acompanhante } \\
\cline { 3 - 6 } & - rosa & crustáceos & moluscos & peixes & Total \\
\hline $1993^{(1)}$ & 90,4 & - & 104,1 & $1.197,7$ & $1.301,8$ \\
$1994^{273,6}$ & - & - & $2.986,7$ & $2.986,7$ \\
$1995^{(2)}$ & 198,0 & - & 215,9 & $2.383,0$ & $2.598,9$ \\
$1996^{(3)}$ & 141,5 & - & 211,2 & $2.318,8$ & $2.530,0$ \\
$1997^{(110,0}$ & 96,8 & 478,5 & $2.228,3$ & $2.803,6$ \\
\hline Média & 162,7 & 19,4 & 201,9 & $2.222,9$ & $2.444,2$ \\
\hline
\end{tabular}

Observações: (1) - com exclusão dos meses de março a maio; (2) - com exclusão dos meses de fevereiro a abril; (3) - com exclusão dos meses de março e abril. Fonte: Instituto Brasileiro do Meio Ambiente e dos Recursos Naturais Renováveis / Superintendência do Estado do Rio de Janeiro (IBAMA / SUPES - RJ).

Tabela II - Relações entre capturas desembarcadas do camarão-rosa e da fauna acompanhante, resultantes de pescarias ao longo da costa do estado do Rio de Janeiro (Brasil), nos anos 1993 - 1997.

\begin{tabular}{c|c|c|c|c|c}
\hline \multirow{2}{*}{ Anos } & \multicolumn{5}{|c}{ Relações entre capturas desembarcadas $(\mathrm{t})$} \\
\cline { 2 - 6 } & camarão & \multicolumn{4}{|c}{ fauna acompanhante } \\
\cline { 3 - 6 } & - rosa & crustáceos & moluscos & peixes & Total \\
\hline 1993 & 1,0 & - & 1,2 & 13,2 & 14,4 \\
1994 & 1,0 & - & - & 10,9 & 10,9 \\
1995 & 1,0 & - & 1,1 & 12,0 & 13,1 \\
1996 & 1,0 & - & 1,5 & 16,4 & 17,9 \\
1997 & 1,0 & 0,9 & 4,3 & 20,3 & 25,5 \\
\hline Médias & 1,0 & 0,1 & 1,2 & 13,7 & 15,0 \\
\hline
\end{tabular}

Observação: desembarques docamarão-rosa: desembarques da fauna acompanhante.
A partir dos registros do esforço de pesca da frota, nos anos considerados (Tabela VI), nos foi possível calcular a produtividade das pescarias do camarão-rosa e de sua fauna acompanhante (Tabela VII).

Tabela III - Capturas desembarcadas mensais do camarãorosa e da fauna acompanhante, resultantes de pescarias ao longo da costa do estado do Rio de Janeiro (Brasil), nos anos de 1993 - 1997.

\begin{tabular}{|c|c|c|c|c|c|c|c|}
\hline \multirow{2}{*}{ Meses } & \multicolumn{5}{|c|}{ Anos } & \multicolumn{2}{|c|}{ Médias } \\
\hline & 1993 & 1994 & 1995 & 1996 & 1997 & $t$ & $\%$ \\
\hline \multicolumn{8}{|c|}{ Desembarque do camarão-rosa $(\mathrm{t})$} \\
\hline I & 1,5 & 11,0 & 13,2 & 7,0 & 4,1 & 7,4 & 4,5 \\
\hline II & 2,1 & 4,0 & - & 5,7 & 2,1 & 2,8 & 1,7 \\
\hline III & - & 3,1 & - & - & 0,1 & 0,6 & 0,4 \\
\hline IV & - & 3,4 & - & - & 0,4 & 0,8 & 0,5 \\
\hline V & - & 29,4 & 9,2 & 9,0 & 10,0 & 11,5 & 7,1 \\
\hline VI & 4,6 & 54,8 & 41,1 & 7,0 & 20,9 & 25,7 & 15,8 \\
\hline VII & 9,6 & 47,5 & 44,1 & 15,2 & 23,0 & 27,9 & 17,1 \\
\hline VIII & 10,7 & 38,7 & 26,4 & 30,4 & 18,4 & 24,9 & 15,3 \\
\hline IX & 12,3 & 34,8 & 11,7 & 23,7 & 17,8 & 20,1 & 12,3 \\
\hline$x$ & 27,1 & 30,9 & 24,2 & 20,8 & 11,6 & 22,9 & 14,1 \\
\hline XI & 13,4 & 12,3 & 17,9 & 12,8 & 1,2 & 11,5 & 7,1 \\
\hline XII & 9,1 & 3,6 & 10,3 & 10,0 & 0,6 & 6,7 & 4,1 \\
\hline Totais & 90,4 & 273,6 & 198,0 & 141,5 & 110,0 & 162,7 & 100,0 \\
\hline \multicolumn{8}{|c|}{ Desembarque da fauna acompanhante $(t)$} \\
\hline I & 82,5 & 357,9 & 385,9 & 208,9 & 238,3 & 254,7 & 10,4 \\
\hline II & - & 253,2 & - & 305,1 & 286,6 & 169,0 & 6,9 \\
\hline III & - & 328,5 & - & - & 355,5 & 136,8 & 5,6 \\
\hline IV & - & 284,5 & - & - & 337,0 & 124,3 & 5,1 \\
\hline $\mathrm{V}$ & - & 298,9 & 298,6 & 299,5 & 226,9 & 224,8 & 9,2 \\
\hline VI & 82,0 & 254,6 & 331,8 & 97,2 & 286,4 & 210,4 & 8,6 \\
\hline VII & 172,5 & 233,4 & 282,6 & 256,6 & 248,9 & 238,8 & 9,8 \\
\hline VIII & 195,7 & 312,0 & 206,7 & 259,7 & 236,7 & 242,2 & 9,9 \\
\hline IX & 144,8 & 173,6 & 131,8 & 280,2 & 316,4 & 209,4 & 8,6 \\
\hline$x$ & 242,3 & 230,2 & 300,4 & 279,0 & 227,7 & 255,9 & 10,5 \\
\hline XI & 157,7 & 169,8 & 350,3 & 259,2 & 30,2 & 193,5 & 7,9 \\
\hline XII & 224,3 & 90,0 & 310,7 & 284,7 & 12,8 & 184,5 & 7,5 \\
\hline Totais & $1.301,8$ & $2.986,7$ & $2.598,9$ & $2.530,0$ & $2.803,6$ & $2.444,2$ & 100,0 \\
\hline
\end{tabular}

Observação: houve omissão de registros das capturas do camarãorosa, nos meses de fevereiro a abril/maio, burlandoépocas de defeso. 
Tabela IV - Relações entre as capturas mensais desembarcadas do camarão-rosa e da fauna acompanhante $(\mathrm{t})$, resultantes de pescarias ao longo da costa do estado do Rio de Janeiro (Brasil), nos anos de 1993 - 1997.

\begin{tabular}{c|c|c|c|c|c|c}
\hline \multirow{2}{*}{ Meses } & \multicolumn{5}{|c|}{ Anos } & \multirow{2}{*}{ Médias } \\
\cline { 2 - 6 } & 1993 & 1994 & 1995 & 1996 & 1997 & \\
\hline I & $1,0: 53,6$ & $1,0: 32,4$ & $1,0: 29,3$ & $1,0: 29,9$ & $1,0: 58,8$ & $1,0: 34,6$ \\
V & - & $1,0: 10,2$ & $1,0: 32,5$ & $1,0: 33,4$ & $1,0: 22,7$ & $1,0: 19,5$ \\
VI & $1,0: 17,7$ & $1,0: 4,6$ & $1,0: 8,1$ & $1,0: 13,9$ & $1,0: 13,7$ & $1,0: 8,2$ \\
VII & $1,0: 18,1$ & $1,0: 4,9$ & $1,0: 6,4$ & $1,0: 16,9$ & $1,0: 10,8$ & $1,0: 8,6$ \\
VIII & $1,0: 18,4$ & $1,0: 8,1$ & $1,0: 7,8$ & $1,0: 8,6$ & $1,0: 12,9$ & $1,0: 9,7$ \\
IX & $1,0: 11,7$ & $1,0: 5,0$ & $1,0: 11,3$ & $1,0: 11,8$ & $1,0: 17,7$ & $1,0: 10,4$ \\
X & $1,0: 8,9$ & $1,0: 7,5$ & $1,0: 12,4$ & $1,0: 13,4$ & $1,0: 19,7$ & $1,0: 11,2$ \\
XI & $1,0: 11,8$ & $1,0: 13,9$ & $1,0: 19,5$ & $1,0: 20,2$ & $1,0: 24,6$ & $1,0: 16,8$ \\
XII & $1,0: 24,6$ & $1,0: 24,9$ & $1,0: 30,1$ & $1,0: 28,6$ & $1,0: 22,9$ & $1,0: 27,5$ \\
\hline Anos & $1,0: 14,4$ & $1,0: 10,9$ & $1,0: 13,1$ & $1,0: 17,9$ & $1,0: 25,5$ & $1,0: 15,0$ \\
\hline
\end{tabular}

Observação: as relações correspondentes aos meses de fevereiro a abril deixaram de ser calculadas, por causa da omissão de registros das capturas do camarão-rosa, em épocas de defeso.

Tabela V - Participação relativa (\%) de grupos e principais espécies da fauna acompanhante do camarão-rosa, nos desembarques resultantes de pescarias ao longo da costa do estado do Rio de Janeiro (Brasil), nos anos de 1993 - 1997.

\begin{tabular}{l|r|l|r}
\hline \multicolumn{2}{l}{$\begin{array}{l}\text { Grupos e espécies da } \\
\text { fauna acompanhante }\end{array}$} & \multicolumn{2}{c}{$\begin{array}{c}\text { Grupos e espécies da } \\
\text { fauna acompanhante (\%) }\end{array}$} \\
\hline Crustáceos & 0,8 & - linguados & 3,8 \\
\hline Moluscos & 8,3 & - maria-mole & 2,7 \\
- lulas & 5,4 & - merluza & 2,6 \\
- polvos & 2,9 & - peixe-sapo & 3,7 \\
\hline Peixes & 90,9 & - tira-vira & 5,7 \\
- arraias & 3,5 & - trilha & 5,6 \\
- cangulo & 3,2 & - viola & 3,3 \\
- castanha & 5,7 & - outros & 24,3 \\
- congro-rosa & 2,5 & - mistura & 21,9 \\
- corvina & 2,4 & TOTAL GERAL & 100,0 \\
\hline
\end{tabular}

Tabela VI - Esforço de pesca $\left(n^{\circ}\right)$ aplicado em pescarias do camarão-rosa e da fauna acompanhante, efetuadas ao longo da costa do estado do Rio de Janeiro (Brasil), nos anos de 1993 - 1997.

\begin{tabular}{c|r|r|r}
\hline \multirow{2}{*}{ Anos } & \multicolumn{3}{|c}{ Unidades do esforço de pesca $\left(\mathrm{n}^{\circ}\right)$} \\
\cline { 2 - 4 } & \multicolumn{1}{c|}{ dias } & \multicolumn{1}{c}{ lances } & \multicolumn{1}{c}{ horas } \\
\hline 1993 & 3.881 & 9.609 & 40.588 \\
1994 & 6.932 & 30.853 & 166.357 \\
1995 & 8.378 & 22.250 & 89.881 \\
1996 & 7.879 & 25.961 & 105.875 \\
1997 & 4.834 & 29.499 & 116.017 \\
\hline Médias & 6.381 & 23.634 & 103.744 \\
\hline
\end{tabular}

Tabela VII - Produtividade das pescarias do camarão-rosa e da fauna acompanhante, considerando desembarques efetuados no estado do Rio de Janeiro (Brasil), resultantes de capturas ao longo de sua costa, nos anos de 1993 - 1997.

\begin{tabular}{l|r|r|r|r|r|r}
\hline \multirow{2}{*}{ Capturas } & \multicolumn{6}{|c}{ Produtividade das pescarias } \\
\cline { 2 - 7 } & 1993 & 1994 & 1995 & 1996 & 1997 & médias \\
\hline Camarão-rosa & 23 & 39 & 24 & 18 & 23 & 25 \\
\hline Fauna & 336 & 431 & 310 & 321 & 580 & 396 \\
acompanhante & - & - & - & - & 20 & 4 \\
crustáceos & - & - & 26 & 27 & 99 & 36 \\
moluscos & 27 & - & 284 & 294 & 461 & 356 \\
peixes & 309 & 431 & 284 & \\
\hline
\end{tabular}

\begin{tabular}{l|r|r|r|r|r|r}
\hline Camarão-rosa & 9 & 9 & 9 & 5 & 4 & 7 \\
\hline Fauna & 136 & 97 & 117 & 97 & 95 & 108 \\
acompanhante & & & & & & \\
crustáceos & - & - & - & - & 3 & 1 \\
moluscos & 11 & - & 10 & 8 & 16 & 9 \\
peixes & 125 & 97 & 107 & 89 & 76 & 99 \\
\hline
\end{tabular}

\begin{tabular}{l|r|r|r|r|r|r}
\hline Camarão-rosa & 2 & 2 & 2 & 1 & 1 & 2 \\
\hline Fauna & 33 & 18 & 29 & 24 & 24 & 26 \\
acompanhante & & & & & & \\
crustáceos & - & - & - & - & 1 & 0 \\
moluscos & 3 & - & 2 & 2 & 4 & 2 \\
peixes & 30 & 18 & 27 & 22 & 19 & 23 \\
\hline
\end{tabular}

\section{RESULTADOS E DISCUSSÃO}

As capturas industriais do camarão-rosa no Estado do Rio de Janeiro (1993 - 1997) mostram tendência decrescente a partir de 1994, o mesmo não se observando com os desembarques da fauna acompanhante; as médias anuais corresponderam a 162,7 t para o camarão-rosa e $2.444,2$ t para o total da fauna acompanhante (Tabeça I). No tocante às relações entre capturas (Tabela II), para cada tonelada capturada do camarão-rosa foram desembarcadas $15,0 \mathrm{t}$ de fauna acompanhante, sendo 0,1 t de crustáceos, 1,2 t de moluscos e 13,7 $t$ de peixes. Convém ressaltar a crescente participação da fauna acompanhante em relação às capturas do camarão-rosa, à medida que estas decrescem, chegando ao máximo de 1,0 t de camarão-rosa : 25,5 t da fauna acompanhante (1997). Daí ser fácil concluir pelo maior aproveitamento da 
fauna acompanhante, em conseqüência da queda de produção do camarão-rosa.

As capturas do camarão-rosa se concentraram nos meses de junho-outubro (fim do outono começo da primavera), perfazendo o total de $74,6 \%$ da produção anual, o mesmo não se observando com os desembarques da fauna acompanhante (Tabela III). Já no tocante à relação camarão-rosa: fauna acompanhante, esta tende a crescer de junho a janeiro, caindo em maio (após o defeso), com o máximo de 1,0 t de camarão-rosa : 34,6 t de fauna acompanhante em janeiro (Tabela IV).

$\mathrm{Na}$ composição dos desembarques em peso da fauna acompanhante, os crustáceos participaram com apenas $0,8 \%$; os moluscos ficaram com $8,3 \%$, com destaque para as lulas; com $90,9 \%$ para os peixes, entre os quais convém assinalar a castanha 5,7\%, o tira-vira 5,7\% e a trilha 5,6\% (Tabela V).

$\mathrm{O}$ esforço de pesca aplicado pela frota camaroneira, em qualquer das suas unidades, mostrou-se com distribuição aleatória no decorrer dos anos (Tabela VI), mas possibilitou os cálculos de relações entre unidades: 1 dia de pesca $=3,7$ lances $=16,3$ horas de arrasto; 1 lance $=4,4$ horas de arrasto.

Quanto à produtividade das pescarias (Tabela VII), as médias encontradas foram as seguintes: camarão-rosa $-25 \mathrm{~kg} /$ dia $=7 \mathrm{~kg} /$ lance $=2 \mathrm{~kg}$ / hora; fauna acompanhante $=396 \mathrm{~kg} / \mathrm{dia}=108 \mathrm{~kg}$ / lance $=26 \mathrm{~kg} /$ hora. Os dados mostram a queda da abundância relativa do camarão-rosa, bem evidente a partir de 1994, o que não se observou com a fauna acompanhante.

\section{CONCLUSÕES}

1. As capturas industriais do camarão-rosa foram decrescentes a partir de 1994, o mesmo não acontecendo com a fauna acompanhante; as médias anuais corresponderam a $162,7 \mathrm{t}$ para o camarão-rosa e a $2.444,2 \mathrm{t}$ para a fauna acompanhante.

2. Para cada tonelada capturada do camarão-rosa foram desembarcadas $15,0 \mathrm{t}$ de fauna acompanhante, sendo 0,1 t de crustáceos, 1,2 t de moluscos e 13,7 $\mathrm{t}$ de peixes.

3. O aproveitamento da fauna acompanhante cresceu com a queda de produção do camarão-rosa.

4. As capturas do camarão-rosa se concentraram nos meses de junho - outubro, perfazendo o total de $74,6 \%$ da produção anual, o mesmo não tendo sido observado com a fauna acompanhante.

5. A relação camarão-rosa: fauna acompanhante cres- ceu de junho a janeiro, com o máximo de 1,0 t de camarão-rosa : 34,6 t de fauna acompanhante.

6. Na composição dos desembarques em peso da fauna acompanhante, os crustáceos participaram com apenas $0,8 \%$, os moluscos com $8,3 \%$ e os peixes com 90,9\%; as espécies mais destacadas foram as lulas, a castanha, o tira-vira e a trilha.

7. As relações entre unidades do esforço de pesca foram as seguintes: 1 dia de pesca $=3,7$ lances $=$ 16,3 horas; 1 lance $=4,4$ horas.

8. As médias da produtividade das pescarias para o camarão-rosa foram $25 \mathrm{~kg} /$ dia $=7 \mathrm{~kg} /$ lance $=2 \mathrm{~kg}$ / hora; para a fauna acompanhante corresponderam a $396 \mathrm{~kg} /$ dia $=108 \mathrm{~kg} /$ lance $=26 \mathrm{~kg} /$ hora .

9. A queda da abundância relativa do camarão-rosa foi bem evidente a partir de 1994, o que não se observou com a fauna acompanhante.

\section{GLOSSÁRIO DE NOMES VULGARES}

Arraias = espécies da ordem Rajiformes; cangulo = Balistes vetula Linnaeus, 1758; castanha = Umbrina canosai Berg, 1895; congro-rosa = Genypterus brasiliensis (Regan, 1903); corvina = Micropogonias furnieri (Desmarest, 1823); linguados = espécies da ordem Heterosomata, principalmente Paralichthys brasiliensis (Ranzani, 1840) e Paralichthys triocellatus (Ribeiro, 1903); lulas = espécies da classe Cephalopoda, principalmente Loligo plei (Blainville, 1823) e Loligo sanpaulensis Brackonieki, 1984; mariamole $=$ Nebris microps Cuvier, 1930; merluza = Merluccius hubbsi Marini, 1933; peixe-sapo (mangangá-liso) = Porichthys porosissimus (Valenciennes, 1837); polvos = espécies da classe Cephalopoda, principalmente Octopus vulgaris (Cuvier, 1797) e Eledone massye Voss, 1964; tira-vira = Percophis brasiliensis Quoy \& Gaimard, 1824; trilha = Mullus argentinae Hubbs \& Marini, 1933; viola $=$ Rhinobatos percellens (Walbaun, 1792).

\section{REFERÊNCIAS BIBLIOGRÁFICAS}

D'Incao, F.; Valentini, H. \& Rodrigues, L. F. Relatório da Reunião Técnica de Avaliação das Pescarias de Camarões das Regiões Sudeste e Sul do Brasil. Itajaí (SC), de 06 a 10 de outubro de 1995. IBAMA/Centro de Pesquisa e Extensão Pesqueira das Regiões Sudeste e Sul do Brasil, [16] p., Itajaí, 1995.

Kotas, J.E. "By-catch" de camarão-rosa e pescaria em Santa Catarina. Trabalho apresentado no XI Encon- 
tro Brasileiro de Ictiologia - PUC/Campinas. Texto datilografado, 61 p., Itajaí, 1995.

Paiva, M.P. Recursos pesqueiros estuarinos e marinhos do Brasil. Edições UFC, 268 p., Fortaleza, 1997.

Pérez Farfante, I. \& Kensley, B. Penaeoid and sergestoid shrimps and prawns of the world (keys and diagnoses for the families and genera). Muséum National d'Histoire Naturelle, 233 p., Paris, 1997.

Vianna, M. Análise de populações de peixes teleósteos acompanhantes da pesca de arrasto do camarãorosa (Penaeus brasiliensis e P. paulensis) em Ubatuba (SP): captura, crescimento e mortalidade. Tese de Doutorado, Universidade Federal de São Carlos, X + 114 p., São Carlos, 1998.

Zenger JR., H. H. \& Agnes, J.L. Distribuição do camarão-rosa (Penaeus brasiliensis e Penaeus paulensis) ao longo da costa sudeste e sul do Brasil. PDP, ser. Doc. Téc., Brasília, n. 21, p. 1-135, 1977. 